\title{
DISEÑO Y FORMULACIÓN DE UN CHAMPÚ A BASE DE EXTRACTO ALCOHÓLICO DE Urtica urens L. PARA SU APLICACIÓN CONTRA LA CAÍDA DEL CABELLO
}

\author{
Jhonnel Samaniego Joaquin ${ }^{* a}$, César Fuertes Ruitón ${ }^{\mathrm{a}}$
}

\begin{abstract}
RESUMEN
Se diseñó y formuló un champú conteniendo el extracto alcohólico de Urtica urens L. utilizando un método analítico, experimental y comparativo. Se utilizó las hojas de la planta (Formulación champú) y conejos blancos, raza neozelandesa machos (Ensayo irritación para evaluación biológica de productos sanitarios). Formaron parte del presente estudio personas diagnosticadas con caída del cabello de origen no patológico, en número de 10 personas que fueron evaluados por un profesional médico, por espacio de 21 días. Se sometieron a observación microscópica los cabellos caídos a diario para evaluar si existe aumento del diámetro del cabello comparándolos al inicio y al final del estudio. Se obtuvo el recuento de cabellos caídos por día durante todo el tratamiento. Utilizando el método de recuento de cabellos estandarizado por 60 segundos. Se obtuvieron como resultados que el porcentaje de reducción de caída del cabello fue de $56,8 \%$ para el grupo de personas que utilizaron el champú a una concentración al 2\% y 32,1\% para el grupo de personas que utilizaron el champú a una concentración al 5\%. Se compararon los promedios de diámetro de cabellos caídos y evaluación de recuento de pelos caídos por día, utilizando el método de recuento de cabellos estandarizado por 60 segundos. Se pudo diseñar y formular un champú conteniendo extracto alcohólico de Urtica urens L.; demostrando su efectividad en el tratamiento contra la caída del cabello logrando una mejora mayor al 50\% y además se determinó que no produce irritabilidad dérmica.
\end{abstract}

Palabras clave: Caída del cabello, champú, extracto alcohólico de Urtica urens L., recuento de cabellos estandarizado por 60 segundos.

\section{DESIGN AND FORMULATION OF A SHAMPOO BASED ALCOHOLIC EXTRACT OF Urtica urens L. FOR APPLICATION AGAINST HAIR LOSS.}

\begin{abstract}
I designed and formulated a shampoo containing the alcoholic extract of Urtica urens L. using an analytical, experimental and comparative method. white rabbits, New Zealand breed

a Facultad de Farmacia y Bioquímica, Universidad Nacional Mayor de San Marcos, Jr. Puno 1002. Jardín
Botánico. Lima. Perú. jhonnel28@hotmail.com
\end{abstract}


males (irritation test for biological evaluation of medical devices) were used. They were part of this study diagnosed with hair loss non-pathological origin; numbering 10 people were evaluated by a medical professional, for 21 days. They were subjected to microscopic observation fallen hair daily to assess whether there is increased hair diameter compared to the beginning and end of the study. fallen hair count per day throughout the treatment was obtained. Using the method of standardized hair count by 60 seconds. They were obtained as results that the percentage of hair loss reduction was $56.8 \%$ for the group of people who used the shampoo at a concentration of $2 \%$ and $32.1 \%$ for the group of people using a shampoo $5 \%$ concentration. the average diameter of fallen hair count and evaluation of fallen hairs per day were compared, using the hair counting method standardized for 60 seconds. It was able to design and make a shampoo containing alcoholic extract of Urtica urens L.; showing its effectiveness in treating hair loss achieving greater than 50\% improvement and determined not produce skin irritability.

Key words: Hair, shampoo, alcoholic extract of Urtica urens L., standardized hair count by 60 seconds.

\section{INTRODUCCIÓN}

Aunque la pérdida del cabello no es una enfermedad debilitante o que amenaza la vida, el mismo pensamiento de convertirse calvo puede conducir al estrés emocional y experiencia traumática para aquellos que sufren de excesiva pérdida de pelo. Muchos intentarán cualquier cosa y todo para evitar la caida de sus cabellos. Los que sufren de pérdida de cabello gastan miles de millones de dólares anualmente en remedios que van desde drogas, vitaminas a tónicos especiales y champús. Tratamientos convencionales de adelgazamiento del cabello incluye terapia de medicamentos y trasplante de cabello. Minoxidil y Propecia (Finasteride) son los únicos dos medicamentos aprobados por la FDA para el crecimiento del cabello en los hombres. Minoxidil es el única droga disponible para mujeres con alopecia androgenética. Se ha demostrado que estos fármacos muestran resultados cuando se presenta calvicie en el vertice del cuero cabelludo. Aunque estos fármacos son eficaces, muchos desconfían de sus efectos a largo plazo, aún desconocido y de sus posibles efectos secundarios. Esto ha llevado a incrementar el interés en remedios como la medicina herbaria ${ }^{1}$.

Según Charlet ${ }^{2}$, los elementos constitutivos del pelo son aminoácidos fisiológicos especialmente la cistina que se sintetiza en su raíz para formar cadenas de queratina. El enlace peptídico de los aminoácidos da origen a la estructura en espiral de las fibras de queratina; se realizó un estudio sobre los aminoácidos presentes en Urtica urens L. encontrándose la presencia de cisteina, la determinación fue realizada en diferentes estados de vegetación de la planta ${ }^{3}$.

El estudio tuvo como objetivo diseñar y formular el champú conteniendo el extracto alcohólico de Urtica urens L. 


\section{PARTE EXPERIMENTAL}

Recolección y certificación taxonómica de la especie para el estudio: como material biológico se utilizaron hojas secas de Urtica urens L. procedentes de Huaraz, se recolectaron de la localidad de Mancos, distrito de Yungay, provincia de Yungay en el departamento de Ancash. Época de floración en julio del 2012. La clasificación taxonómica se realizó en el Museo de Historia Natural de la UNMSM. Constancia N¹77-USM-2012.

\section{Molienda, estabilización y desecación de la planta}

Las hojas de la especie se lavaron con agua destilada y fueron secadas en un horno con circulación de aire a una temperatura aproximada de $37^{\circ} \mathrm{C}$. Luego fueron trituradas en un molino de cuchillos hasta obtener un polvo fino y posteriormente se almacenaron en botellas de vidrio a temperatura ambiente ${ }^{4}$.

\section{Preparación del extracto alcohólico}

Las hojas en polvo $(5 \mathrm{~kg})$ fueron sometidas a extracciones continuas en maceración con etanol al $70 \%{ }^{4}$, a temperatura de $25{ }^{\circ} \mathrm{C}$ en un recipiente de vidrio cerrado y de color ambar, por siete días; posteriormente se separó la solución alcohólica del marco utilizando papel de filtro Whatman $\mathrm{N}^{\circ} 1$. Los filtrados se recolectaron en un solo recipiente y se concentraron hasta sequedad a presión reducida en un rotavapor, obteniéndose el extracto seco.

\section{Análisis fitoquímico del extracto alcohólico de Urtica urens L.}

Los reactivos usados fueron, ácido clorhídrico concentrado (37\%), limadura de magnesio, reactivo de ninhidrina, reactivo de Dragendorff, alcohol etílico (70\%) y agua destilada.

El extracto fue analizado utilizando reacciones químicas de coloración para determinar flavonoides, taninos y compuestos fenólicos ${ }^{5}$.

Se utilizó el extracto alcohólico de Urtica urens L. y analizado según reacción Shinoda; para identificar isoflavonas y corroborar el comportamiento fluorescente por cromatografía en placa de sílice, para observar una coloración purpura sin cambios con vapores de amoniaco ${ }^{5}$.

\section{Método de preparación del champú}

El champú fue elaborado de acuerdo al método señalado en Simmons ${ }^{6}$.

Se incorporó la materia activa detergente con la mitad de agua destilada hasta completa disolución para luego agregar los conservantes con agitación moderada sin formar burbujas de aire; luego se adicionó el agente suavizante y aditivo especial y se agitó hasta homogeneizar, en otro recipiente se disolvió el extracto de ortiga con la otra mitad de agua y enseguida se agregó el colorante hasta completa homogeneización para luego adicionar esta mezcla al preparado anterior que contiene la materia activa detergente y una vez obtenida la mezcla total se agregó el espesante con agitación continua para obtener la viscosidad deseada. Se elaboró el champú a dos concentraciones de extracto de Urtica urens L. al 2\% y al 5\% (tabla 1); para su aplicación contra la caída del cabello, de agradable fragancia con una presentación en un envase de $300 \mathrm{~mL}$, de fácil manipulación por su diseño. 
Tabla 1. Composición del champú en sus dos concentraciones de extracto de Urtica urens L.

\begin{tabular}{|l|l|l|}
\hline \multicolumn{1}{|c|}{ Materia prima } & $\begin{array}{c}\text { Champú } \\
\text { al 2\% }\end{array}$ & $\begin{array}{c}\text { Champú } \\
\text { al 5\% }\end{array}$ \\
\hline Materia activa detergente & $25,000 \%$ & $25,000 \%$ \\
\hline Suavizantes & $5,500 \%$ & $5,500 \%$ \\
\hline Colorante & $0,002 \%$ & $0,002 \%$ \\
\hline Esencias & $0,500 \%$ & $0,500 \%$ \\
\hline Conservantes & $0,200 \%$ & $0,200 \%$ \\
\hline Extracto Urtica urens L. & $2,000 \%$ & $5,000 \%$ \\
\hline Espesante & $0,300 \%$ & $0,300 \%$ \\
\hline Agua destilada & $66,498 \%$ & $63,498 \%$ \\
\hline Total & $100.000 \%$ & $100.000 \%$ \\
\hline
\end{tabular}

Seguridad: En el ensayo de irritabilidad dérmica se utilizaron animales de experimentación: 5 Oryctolagus cuniculus (conejos), raza neozelandesa, machos, de aproximadamente $2 \mathrm{~kg}$ de peso, correctamente alimentados. Los animales fueron adquiridos del cepario del Instituto Nacional de Salud - Chorrillos, 20 personas sanas con caída del cabello de origen cosmético no alopécico

\section{a. Ensayo de irritación y sensibilización cutánea}

Se realizó la evaluación del potencial del producto para producir irritación cutánea en un modelo animal (conejo). El material de ensayo fue el champú con el extracto de Urtica urens L. a dos concentraciones, 2 y $5 \%$, y un control positivo con el champú sin extracto. Para la preparación de los animales de experimentación se utilizaron cinco conejos albinos adultos y sanos machos pertenecientes a una única cepa, con la piel intacta y sana; a los cuales se les rasuró el pelo 12 horas antes de comenzar el ensayo en los dorsos en un tamaño aproximadamente de 10 x $15 \mathrm{~cm}$. La aplicación de la muestra se realizó con $0,5 \mathrm{~g}$ del champú sobre la piel y se cubrieron los lugares de aplicación con un apósito (parche de gasa) y luego se vendaron estos lugares por 4 horas. Al final del tiempo de contacto se levantaron los apósitos y se marcaron las posiciones de la zona y se evaluaron los resultados según la NT ISO 10993-10 ${ }^{7}$. En la calificación de la irritación cutánea se empleó el sistema indicado en la tabla 2.

Tabla 2. Sistema de clasificación de reacciones cutáneas

\begin{tabular}{|cc|}
\hline Reacción dérmica & Puntuación \\
\hline Formación de eritema y escara & \\
Ausencia de eritema & 0 \\
Eritema muy leve (apenas perceptible) & 1 \\
Eritema bien definido & 2 \\
Eritema moderado & 3 \\
Eritema grave a formación de escara que hace imposible la graduación & 4 \\
$\quad$ del eritema & \\
Formación de edema & \\
Ausencia de edema & 0 \\
Edema muy leve (apenas perceptible) & 1 \\
Edema bien definido (bordes de la zona bien delimitados por una & 2 \\
pápula netamente perceptible) & 3 \\
Edema moderado (elevación de la pápula aproximadamente 1 mm) & 4 \\
\hline zdema grave (elevación de la pápula mayor de 1 mm y rebasando la exposición) & 8 \\
\hline Puntuación máxima posible para la irritación & \\
\hline
\end{tabular}


El índice de irritación primaria o acumulativa se caracteriza por el número (puntuación) y la descripción (categoría de respuesta) dados en la tabla 3.

Tabla 3. Categorías del índice de irritación primaria o acumulativa en un conejo

\begin{tabular}{|cc|}
\hline Puntuación media & Categoría de la respuesta \\
\hline 0 a 0,4 & Insignificante \\
0,5 a 1,9 & Leve \\
2,0 a 4,9 & Moderado \\
5 a 8 & Grave \\
\hline
\end{tabular}

\section{b. Diseño Experimental para determinar la actividad del extracto alcohólico de Urtica urens $\mathrm{L}$. en el cabello}

Se solicitó la autorización del estudio al Instituto de Ética de la Unidad Académica de la Facultad de Medicina UNMSM.

Criterios de Inclusión

- Personas del sexo masculino a quienes se les ha diagnosticado caída del cabello de origen no patológico, evaluadas por un profesional médico dermatólogo.

- Criterios de Exclusión

- Personas que hayan recibido o reciban tratamiento capilar.

- Diagnosticadas con alopecia.

- Personas con alguna de estas enfermedades: diabetes, psoriasis, neurodermatitis o trastornos en la función tiroidea.

- Personas que hayan tomado o toman hormonas, antidepresivos o citostaticos.

- Personas que hayan usado o usan trenzas, tintes, permanente o se realicen el secado del cabello con aire caliente

- $\quad$ Personas mayores de 40 y menores de 20 años.

Estos criterios fueron evaluados mediante la realización de un examen médico previo y se determinó que los pacientes estában sanos y no presentaron signos de tener alopecia; además de realizarles una entrevista y una encuesta con las preguntas necesarias para determinar que sean las óptimas para los fines del estudio.

- Se trabajó con dos grupos de trabajo: A con champú con extracto al $2 \%$ y B con champú con extracto al $5 \%$, con 10 integrantes en cada uno de ellos y fueron tratados por espacio de 21 días.

- Se sometieron a observación microscópica los cabellos perdidos por los dos grupos, teniendo en cuenta su morfología al inicio y al final del estudio.

\section{Determinación cuantitativa de pelos caídos}

1. Se obtuvo el recuento de pelos caídos por día durante todo el tratamiento. Utilizando el método de recuento de cabellos estandarizado por 60 segundos; el cual se realizó peinándose hacia adelante en las mañanas por 60 segundos antes de lavarse el cabello y recogiendo los pelos caídos sobre una toalla de color contrastante para que los pelos puedan ser visualizadas adecuadamente. Los pelos rotos o fragmentados fueron descontados del estudio ${ }^{8}$. 
2. El diámetro del pelo al inicio y al final del tratamiento (visto al microscopio óptico en un aumento de $10 \mathrm{X}$ ).

3. Luego se aplicó el champú una vez al día.

\section{RESULTADOS Y DISCUSIÓN}

En el análisis fitoquímico realizado al extracto alcohólico se comprobó la presencia de los compuestos indicados en la tabla 4.

Tabla 4. Ensayo fitoquímico del extracto alcohólico de las hojas de Urtica urens L.

\begin{tabular}{|ccc|}
\hline Reacciones & Metabolitos secundarios & $\begin{array}{c}\text { Estimación de } \\
\text { componentes }\end{array}$ \\
\hline Shinoda & $\begin{array}{c}\text { Flavonoides } \\
\text { (isoflavonas) }\end{array}$ & +++ \\
Ninhidrina & $\begin{array}{c}\text { Aminoácidos } \\
\text { (cistina) }\end{array}$ & +++ \\
$\begin{array}{c}\text { Reacción de } \\
\text { Dragendorff }\end{array}$ & Alcaloides & ++ \\
\hline
\end{tabular}

++ (Regular cantidad); +++ (Mayor cantidad)

En la tabla 5 se puede observar los resultados negativos obtenidos en el ensayo de irritación cutánea con el extracto seco

Tabla 5. Para el extracto seco

\begin{tabular}{|cc|}
\hline Conejo & Puntuación de la respuesta \\
\hline Con extracto seco & 0 \\
Con vehículo del extracto & 0 \\
\hline Total & 0 \\
\hline
\end{tabular}

En la tabla 6 se reportan los resultados sin respuesta obtenidos en el ensayo de irritación cutánea con el champú en sus dos concentraciones

Tabla 6. Para el champú con extracto de Urtica urens L. en sus dos concentraciones

\begin{tabular}{|cc|}
\hline Conejo & Puntuación de la respuesta \\
\hline Con champú al 2\% de extracto & 0 \\
Con champú al 5\% del extracto & 0 \\
Con champú sin extracto & 0 \\
\hline Total & 0 \\
\hline
\end{tabular}


En la tabla 7 se puede observar un resumen de todos los estudios realizados en el presente trabajo, los cuales presentan resultados favorables luego de la utilización del champú con extracto de Urtica urens L. y se comprobó su validez estadística utilizando las pruebas paramétricas como " $t$ ” de student y análisis de varianza (ANOVA) confirmando indicios sobre su uso tradicional ${ }^{1}$.

Tabla 7. Cantidad promedio del número de cabellos caídos luego de utilizar el champú con el extracto de Urtica urens L. (2 y 5\%) y porcentaje de reducción.

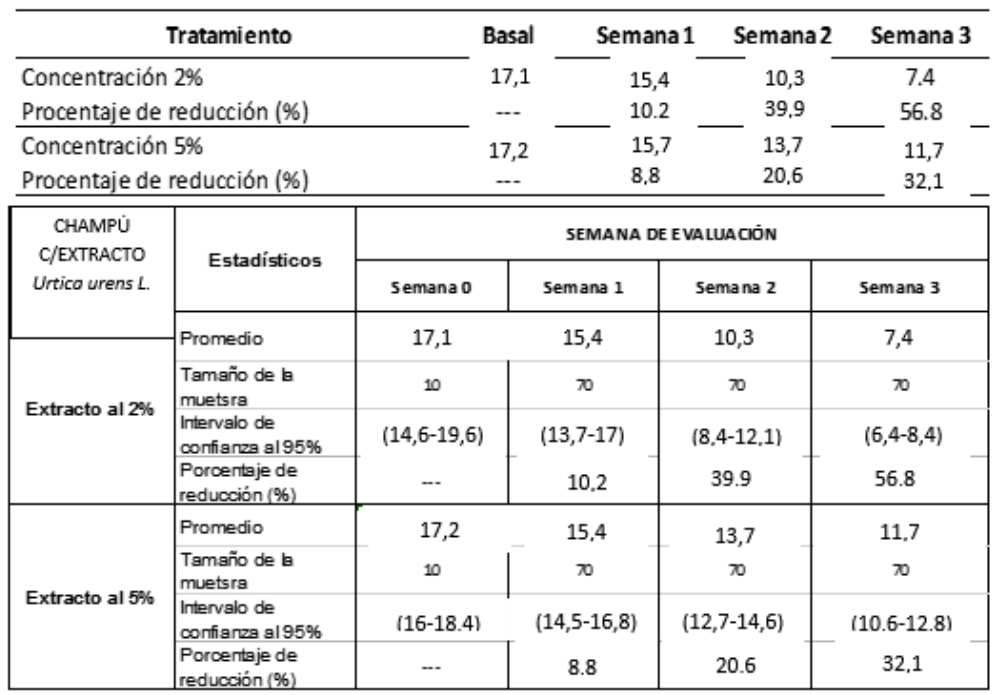

La desiacion esta ndar uada en el calculo del intarvalo deconflanza corresponde a la est ma da a pa rer de la variabilidad entre participa nass. Calda promsdio de partelipantes sa nos sin tratamlento - $5.9(5.5-6.3)$

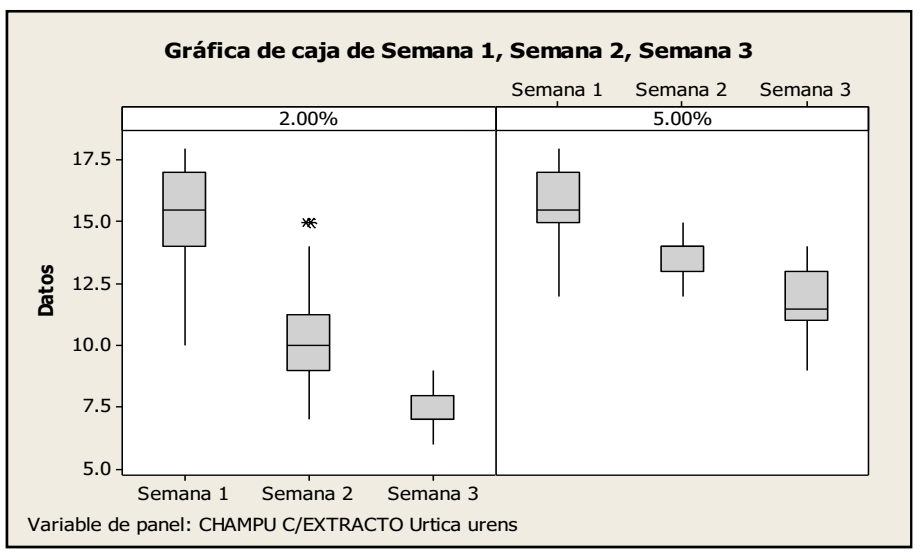

Figura 1. Gráficas de caja al 2 y al 5\% por semanas 


\section{CONCLUSIONES}

Se diseñó y formuló un champú a base de extracto alcohólico de las hojas de Urtica urens L., que según el resultado fitoquímico obtenido presentan isoflavonas, aminoácido como la cistina y alcaloides, encontrándose coincidencias al estudio realizado por Massara en la determinación de flavonoides al extracto de la misma planta.

Se determinó que el champú a base de extracto alcohólico de Urtica urens L. no produce irritación cutánea al evaluarse el potencial del producto en ninguna de sus dos concentraciones efectuado en animales de experimentación según lo estipula la evaluación biológica de productos sanitarios de la norma técnica ISO 10993-10 y así se evaluaron los posibles peligros de contacto con sustancias químicas liberadas de productos sanitarios.

Al evaluar los resultados de la medida de diámetros de cabellos caídos y conteo cantidad de promedio del número de cabellos caídos mediante pruebas estadísticas paramétricas como t de student y análisis de varianza se pudo observar un porcentaje de reducción de caída del cabello mayor en el champú con extracto de Urtica urens L. al 2\% al finalizar las tres semanas de tratamiento.

Se probó la actividad del champú de Urtica urens L. en el tratamiento contra la caída del cabello logrando una mejora mayor de $50 \%$ a la concentración de extracto alcohólico de $2 \%$.

\section{BIBLIOGRAFÍA}

1. Patil SM, Sapkale GN, Surwase US, Bhombe BT. Herbal medicines as an effective therapy in hair loss. Res J Pharm Biol Chem Sci. 2010; 1(2): 773-781

2. Charlet E. Cosmética para farmacéuticos. Zaragoza: Editorial Acribia. 1996.

3. Timofeev PV, Khuzin MT. Amino acid composition of some forage crops and wild grasses. Stud Nauch Rab Univ Druzhby Nar. 1967; 9:38-42.

4. Massara M, Sameh B, Sana B, Zouheir S, Tarek R. Chemical composition, phytochemical constituents, antioxidant and anti-inflamatory activities of Urtica urens L. leaves. Arch Physiol Biochem. 2016; 1-11

5. Lock U. Investigación Fitoquímica. $2^{\mathrm{a}}$ ed. Lima: Univ. Católica. 1994

6. Simmons J. Cosméticos: Formulación, preparación y aplicación. Madrid: Ediciones A. Madrid Vicente. 2000.

7. Comité Europeo de Normalización, Norma Técnica ISO 10993-10, Evaluación Biológica de Productos Sanitarios. Parte 10: Ensayos de Irritación y Sensibilización cutánea. Madrid: Asociación Española de Normalización y Certificación. 2011.

8. Wasko CA, Mackley CL, Sperling LC, Mauger D, Miller JJ. Standardizing the 60-Second Hair Count. Arch Dermatol. 2008, 144(6):759-762.

9. Urtica dioica, Urtica urens (Nettle). Altern Med Rev. 2007; 12 (3):280-284. 